\title{
KRAS-mutant non-small cell lung cancer: recent progress
}

\author{
Chunlu Shu 1, Yuling Liu ${ }^{2, *}$ Cong Zhou ${ }^{3,4, *}$
}

1 State Key Laboratory of Biotherapy and Cancer center, West China Hospital, Sichuan University and Collaborative Innovation Center, Chengdu 610041, China 1; chunlushu@163.com

2 Fenyang College of Shanxi Medical University, 16 College Road, Fenyang, 032200, China

3 Department of Obstetrics and Gynecology, West China Second University Hospital, Sichuan University, Chengdu, China

4 Key Laboratory of Birth Defects and Related Diseases of Women and Children, Ministry of Education, Sichuan University, Chengdu, China

* Correspondence: linger8911@126.com; zhoucongnoi@163.com

Introduction: Rat sarcoma (RAS) genes are the most commonly mutated oncogenes in cancer with mutations of KRAS, NRAS, and HRAS occurring in 30\% of cases. KRAS occur with the highest frequency in the RAS gene family which accounts for $86 \%$ of RAS mutations [1]. KRAS mutation occurs in about $30 \%$ of lung cancer [2], and is the second most frequent genetic variant in non-small cell lung cancer (NSCLC). However, wild-type KRAS protein is small in size and has a smooth surface for the very high affinity of GTP/GDP, making it difficult to fit any small molecule compounds that compete with it. Also, it is tough to find compounds that selectively inhibit KRAS mutations for its wide range of effects. Therefore, there are limited treatment options in NSCLC with KRAS mutation in the past 40 years.

With the discovery of the KRASG12C mutation (glycine to cysteine substitution at position 12) forming a "pocket" on the surface of the KRAS protein for small molecules to bind, which inhibit the activity of KRAS mutants by locking the KRAS ${ }^{\mathrm{G} 12 \mathrm{C}}$ mutant protein in an inactive state, a number of promising direct KRASG12C inhibitors have been developed. For example, Sotorasib and MRTX849 made a breakthrough showing promising anti-tumor effects, accompanied by the failure of many other drug development. However, KRASG12C mutation is only found in approximately $10 \%$ of patients with non-squamous NSCLC. The non-KRASG12C mutation population, which accounts for about $20 \%$ of NSCLC still lack effective treatment. Surprisingly, anti-PD-1/PD-L1 immunotherapy has shown preliminary efficacy and safety in NSCLC with KRAS mutation. Also, there is considerable evidence that the KRAS signaling pathway induces the expression of a variety of immune regulatory factors, thereby forming an immunosuppressive tumor microenvironment from the perspective of mechanism. Preclinical studies have shown that inhibition of KRAS ${ }^{\mathrm{G} 12 \mathrm{C}}$ protein can reversible suppressed immune microenvironments.

In this review, we discuss the latest developments in targeted therapy and immunotherapy for KRAS-mutation NSCLC, aiming to provide direction or enlightenment for the future treatment strategies

Keywords: NSCLC 1; KRAS G12C mutation 2; targeted therapy 3 ; immunotherapy 4

\section{Molecular and clinicopathological features of KRAS-mutant NSCLC}

KRAS encodes a membrane-bound guanosine triphosphatase (GTPase), which play a molecular switch effect by converting GTP molecules into guanosine diphosphate (Guanosine diphosphate, GDP) molecule. KRAS is inactive when bound to guanosine diphosphate (GDP) and active when bound to guanosine triphosphate (GTP). The activation/deactivation process of KRAS involves two regulatory proteins: guanine nucleotide exchange factor (GEF) which promotes the binding of KRAS and GTP to activate including 
SOS (Son of sevenless) protein; and GTPase activating protein (GTPase-activating protein, GAP) which promote GTP combined with KRAS into GDP [3]. When KRAS mutated in codons 12,13 , and 61 , the resulting mutant KRAS protein remains primarily in the active KRAS-GTP state, which disrupts the GTP hydrolysis and guanine exchange rates of RAS proteins. Therefore, it would lead to unregulated oncogenic signaling and tumorigenesis [3].

The vast majority of KRAS mutations (97\%) were found at the 12th or 13th amino acid residues. Common mutations are G12D, G12V, G12C, G12A, and G13D. KRASG12C is one of the most common genetic mutations in NSCLC, with an incidence of $~ 13 \%$ of patients in Western countries and 3\%-5\% of patients in Asia. KRAS mutations occur mainly in male lung adenocarcinoma patients with a history of smoking, with a higher incidence in the elderly [4].

KRAS have co-mutations with other master genes, So KRAS-mutated NSCLC frequently may have genetic heterogeneity rather than only a single KRAS mutation. KRAS mutations do not often co-occur with actionable driver mutations (EGFR, ALK, ROS1, BRAF) [5], but commonly co-mutated with TP53 tumor suppressor gene, serine/threonine kinase 11 (STK11), and kelch-like ECH associated protein 1 (KEAP1) etc. [6]. The pattern of genetic co-mutations varied with different KRAS clusters: (1) KP subgroup (+TP53 mutation); (2) KL subgroups (+ STK11/LKB1 mutation); (3) KC subgroup (+CDKN2A/B inactivation plus low TTF1) [7]. The TP53/KRAS co-mutation resulted in increased expression of PD-L1, an immune-rich microenvironment with TILs, and immunoediting. KL subgroups expressed low levels of PD-L1 and contained few tumor infiltrating lymphocytes (TILs), leading to an inert tumor immune microenvironment. The KC subgroup, which involves $\mathrm{C}+\mathrm{CDKN} 2 \mathrm{~A} / \mathrm{B}$ inactivation plus thyroid transcription factor-1(TTF1) low expression suggesting a role in tumor differentiation, was also associated with mucinous histology and suppressed mTORC1 signaling [8].

\section{The breakthrough in targeted therapy for KRAS ${ }^{\mathrm{G} 12 \mathrm{C}}$ mutant NSCLC}

KRAS has been considered "undruggable" for 40 years because of its unique molecular characteristics until the discovery of a new generation of direct inhibitors of KRASG12C by Ostrem et al.in 2013. Their investigation of the crystal structure of the mutant protein bound to GDP revealed a new pocket beneath the effector binding switch II region, which was not apparent in previous models of RAS. The discovery of this new pocket allowed for the direct targeting of KRAS [9]. Irreversible small molecule inhibitors such as AMG510 and MRTX849 that could target the mutant KRASG12C protein by covalently binding to the mutant cysteine residue have demonstrated activity in early clinical studies.

\section{Sotorasib}

Sotorasib is a small molecule that irreversibly and selectively binds to the mutant C12 in a small pocket (P2) on the KRAS ${ }^{G 12 C}$ protein2, which can lock the KRAS G12C mutant protein in an inactive state, preventing oncogenic signaling without affecting wild-type KRAS[10].

Recently, the clinical researches involve Sotorasib have shown amazing efficacy in patients with KRASG12C mutations. Hong DS presented a phase $1 / 2$ study evaluating the safety, tolerance, Pharmacokinetic (PK), and efficacy of Sotorasib in subjects with solid tumors with a specific KRAS mutation (CodeBreak 100: NCT03600883) at European Society of Medical Oncology 2020 Virtual Congress, showing a favorable safety profile of Sotorasib monotherapy. PK analyses demonstrated that the half-life is approximately 5.5 hours, and brief exposure to Sotorasib $(960 \mathrm{mg})$ is expected to completely inhibit KRASG12C throughout the dosing interval [11]. 
Meanwhile, in phase $1 / 2$, Sotorasib showed encouraging anticancer activity in metastatic NSCLC, CRC, and other tumor types previously treated, with a median of 3 (range: 0-11) previous lines of anticancer therapy. For NSCLC patients( $\mathrm{N}=59)$, ORR was $32 \%$ and disease control rate (DCR) was $88 \%$, with median PFS of 6.3 months; for CRC patients (N=42), ORR was $7 \%$ and disease control rate (DCR) was 74\%, with Median PFS of 4 months; for other tumor Types (N=28), ORR was $14 \%$ and disease control rate (DCR) was $75 \%$.

Phase 2 of the study was published in the New England journal in 2020 by Hong Ds et al. .A total of 126 patients with locally advanced or metastatic NSCLC with the KRASG12C mutation were enrolled from 11 countries, of which $81 \%$ of patients had previously received platinum-based chemotherapy and PD-1/ L1 inhibitors. Sotorasib was orally administered at $960 \mathrm{mg}$ once daily until disease progression. The efficacy and safety for patients with metastatic NSCLC $(\mathrm{N}=124)$ who received Sotorasib is promising: ORR was $37.1 \%$ and disease control rate (DCR) was $80.6 \%$. In these patients with NSCLC, median duration of response was 10.0 months, median time to objective response was 1.4 months, and the median progression-free survival (PFS) was 6.8 months for sotorasib. Treatmentrelated adverse events (TRAEs) were generally mild and manageable. Grade 3 and 4 TRAEs were reported in $19.8 \%$ and $0.8 \%$ of patients, respectively, and no fatal TRAEs were reported [12].

Brain metastasis are very common in lung adenocarcinoma. The difference in the efficacy of small molecule drugs between people with brain metastases and non-brain metastases has been the focus of our attention. According to the data disclosed at the World Lung Cancer Congress in September 2021, the exploratory of brain metastasis subgroup in CodeBreak 100 indicated patients acchieved confirmed tumor remission and OS benefit. The disease control rate DCR was 77.5\%, median PFS was 5.3m (2.7,9.3), median OS was $8.3 \mathrm{~m}(7.3,12.5)$ in patients with brain metastases, while disease control rate DCR was $84.1 \%$, median PFS was 6.7m $(5.3,8.2)$, median OS was $13.6 \mathrm{~m}(10.0, \mathrm{NE})$ in patients with non-brain metastases, respectively. The safety of the two groups is equivalent, with a $20 \%$ (8/40) class 3 TRAEs in the brain metastasis group and 19\% (26/134) in the non-brain metastasis group. Additionally, there were no fatal TRAEs [13]

The KRAS gene is heterogeneous and is often accompanied by different co-mutation genes. It is unclear whether the Sotorasib has the same effect on people with different comutations. Exploratory analysis prensented by Ferdinandos Skoulidis in American Oncology Annual Meeting 2021 from CodeBreak 100 of the subgroups found that different PD-L1 expressions and TMB or KRAS co-mutatant have different tumor responses. Compared with the population with PD-L1 expression, PD-L1 negative patients have relatively higher ORR. Meanwhile, regardless of whether the TMB is high $(\geq 10 \mathrm{mut} / \mathrm{mb})$ or $\operatorname{low}(<$ $10 \mathrm{mut} / \mathrm{mb})$, the ORRs of the two groups are similar. Patients co-mutatant with TP53、 STK11and KEAP1 mutations have different tumor response, while the benefit was relatively small in the combined KEAP1 mutant group. Further analysis of ORR, PFS and OS in patients with co-mutation of STK11 and KEAP1showed improved efficacy with Sotorasib in the STK11-mutant group with concurrent wild-type KEAP1. The median PFS was $11 \mathrm{~m}(2.8, \mathrm{NE})$ and the median OS was $15.3 \mathrm{~m}(4.8, \mathrm{NE})$ whereas the KEAP1-mutant groups seemed to benefit little[14].

Drug resistance is a problem that targeted therapy drugs have to face, and the discovery of drug resistance mechanisms is critical to the further development of drugs. Ferdinandos Skoulidis presented the exploratory analysis of the resistance mechanism of Sotorasib in the treatment of KRAS p.G12C-mutated NSCLC at the World Lung Cancer Congress in September 2021. Gene mapping analysis of baseline tissues revealed that the clinical response patterns varied by baseline co-mutantion. Co-mutation of KEAP1 was associated with early progression (patient progression PFS $<3$ months). which are consistent with the poor prognosis in these patients. Cell cycle (14/27) and WNT pathway $(12 / 24)$ may be 
associated with late progression (patient progression PFS $>3$ months), providing an opportunity to co-treat patients with these co-mutation patterns. RTK showed no association with early or late progression, a result that warrants further study[15].

Furthermore, the global phase 3 trial, CodeBreak 200, comparing Sotorasib with docetaxel in patients with KRASG12C mutated NSCLC is ongoing.

\section{MRTX849}

Another KRASG12C inhibitor under development is adagrasib (MRTX849), a covalent KRASG12C inhibitor that irreversibly and selectively binds to KRASG12C, leaving it in an inactive GDP bound state, and combine it with the Switch II pocket.

According to the data disclosed in a phase 1/2 Study (KRYSTAL-1;NCT03785249), adagrasib showed a favorable safety profile and significant clinical activity in heavily pretreated patients. 18 NSCLC patients from Phase $1 / 1 \mathrm{~b}$ and 51 from Phase $1 / 1 \mathrm{~b}$ and 2 received a $600 \mathrm{mg}$ BID dose adagrasib until disease progression. Analysis showed that ORR was $45 \%$ and disease control rate (DCR) was $96 \%$. The median duration of response was 8.2 months, and median time to objective response was 1.5 months. As far as safety was concerned, 1 patient had grade 5 treatment-related pneumonia. Exploratory analysis showed that patients with STK11 mutations had a higher ORR (64\%). Analysis of immune transcripts before and after Adagrasib treatment revealed that Adagrasib may recruit T cells into tumors and reverse STK11-mediated immunosuppression, but a large number of samples are still required for verification[16].

Furthermore, a phase II study (KRYSTAL-7) of Adagrasib in combination with Pembro for newly treated NSCLC patients with KRASG12C mutations who cannot be treated locally or with metastases is ongoing.

This section may be divided by subheadings. It should provide a concise and precise description of the experimental results, their interpretation, as well as the experimental conclusions that can be drawn.

Table 1. Efficacy data of KRAS ${ }^{\mathrm{G} 12 \mathrm{C}}$ inhibitors

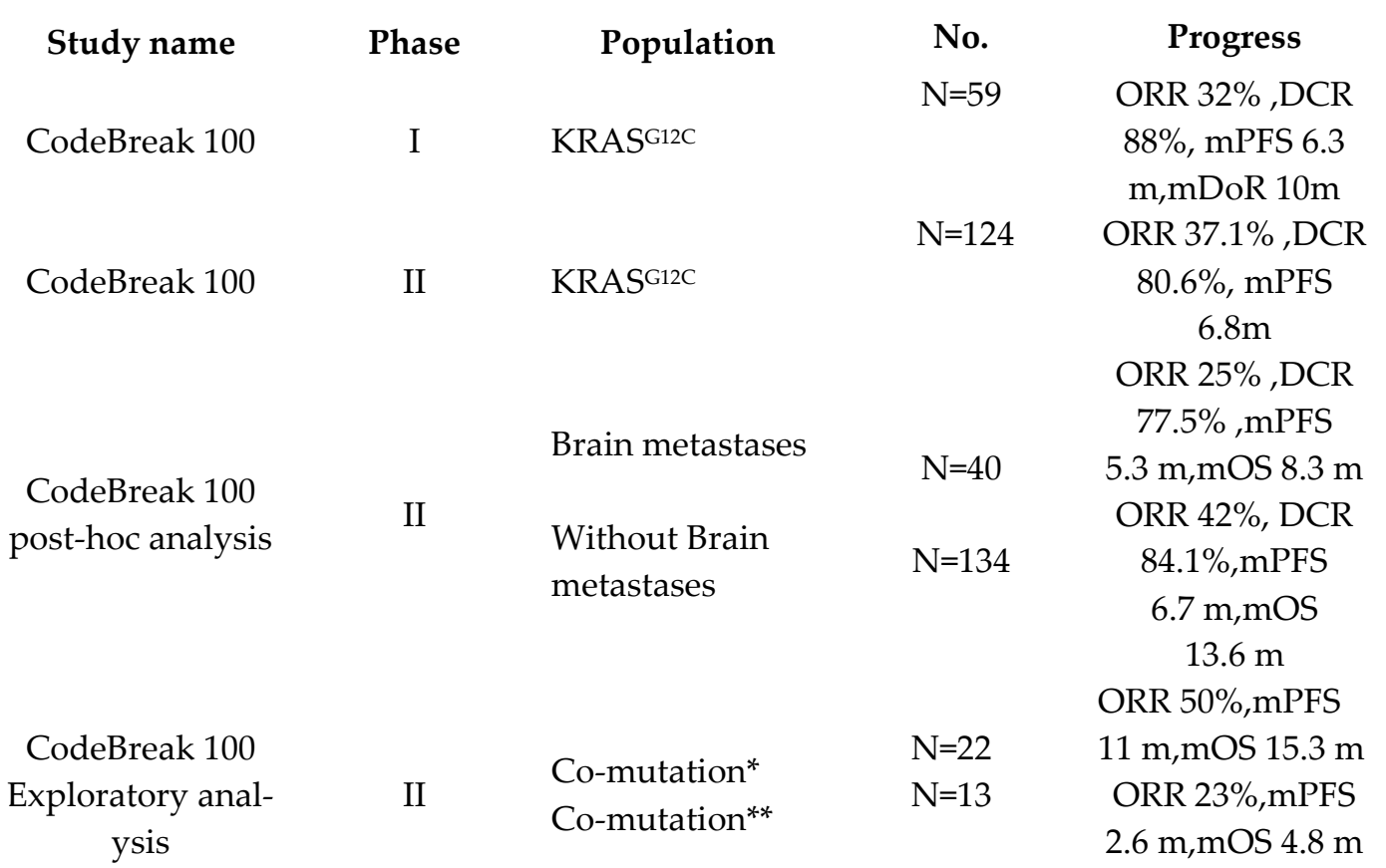




\begin{tabular}{|c|c|c|c|c|}
\hline \multirow[b]{2}{*}{ KRYSTAL-1 } & \multirow[b]{2}{*}{ I } & KRASG12C & $\mathrm{N}=17$ & $\begin{array}{c}\text { ORR } 40 \%, \text { DCR } \\
91.6 \%\end{array}$ \\
\hline & & KRASG12C & $\mathrm{N}=79$ & $\begin{array}{c}\text { ORR } 45 \%, \text { DCR } \\
96 \%, \mathrm{mDoR} 8.2 \mathrm{~m}\end{array}$ \\
\hline KRYSTAL-1 & $\mathrm{I} / \mathrm{II}$ & Co-mutation\# & $\mathrm{N}=14$ & ORR 64\% \\
\hline
\end{tabular}

\section{The Progress in immunotherapy of KRAS mutant NSCLC}

\subsection{Anti-PD-L(1) single agent}

A number of published research data have found that people with KRAS mutations can benefit from immune monotherapy. A meta-analysis including 9 studies with 1,716 cases of NSCLC, 694 cases of KRAS mutations, and 1,022 cases of KRAS wild-type showed that among patients receiving immune monotherapy, people with KRAS gene mutations can benefit more (mutant vs wild type ORR HR: 1.51 (95\%:1.17-1.96)) [17]. In the Checkmate057 study, 62 patients with KRAS mutations benefited more from Nivolumab treatment than chemotherapy ( mOS HR $0.52(95 \% \mathrm{CI} 0.29 \sim 0.95)$ ). In the OAK study, 59 patients with KRAS mutations tended to benefit from Atezolizumab treatment ( mOS HR $0.71(95 \%$ CI $0.38 \sim 1.35)$ ) [18]. In KEYNOTE-042 study, 301 patients, 69 of which possessed KRAS mutations while 29 possessed KRASG12C mutations were analyzed. Compared with KRAS wild-type patients, Pembro significantly prolonged OS in patients with KRAS mutations (HR 0.86 (95\% CI: 0.63-1.18) vs HR 0.42 (95\% CI: 0.22-0.81)). Meanwhile, KRASG12C mutation patients seem to benefit more from OS: HR 0.28 (95\%CI: 0.09-0.86); it should be noted that the patients enrolled in this study are all people with PD-L1 greater than $1 \%$ [19].The IMMUNTRGET study included 246 NSCLC patients with KRAS mutations that received immune monotherapy. The ORR was $26 \%$, the median PFS was 3.2 months (95\%: 2.7-4.5), and the median OS was 13.5 months (95\%): 9.4-15.6), which suggests that people with KRAS mutations can benefit from immunotherapy [20]. For people with KRAS mutations, more large-scale studies are needed to prove that immunotherapy brings survival benefits.

\subsection{Anti-PD-L(1) combined chemotherapy}

About the immune combination therapy for people with KRAS mutation, we summarize the data from the following studies. A Meta-analysis of 6 studies showed that for NSCLC patients with KRAS mutation, immunotherapy combined with chemotherapy significantly prolonged OS compared with chemotherapy alone (HR 0.59 [95CI\%:0.49$0.72]) \mathrm{p}<0.00001$ and PFS (HR 0.58 [95CI\%:0.43-0.78]) $\mathrm{p}=0.0003$, and the OS of the population with KRAS mutation is significantly longer than in the KRAS wild-type group $(\mathrm{P}=0.001)$ [21].

In Keynote-189 study, 89 patients with KRAS mutations (37 with a KRASG12C mutation) were analyzed and there was no significant difference in survival between the Pembro combined chemotherapy and chemotherapy with OS HR was 0.79 (95\%CI: 0.45-1.38). Also, for KRAS ${ }^{\mathrm{G} 12 \mathrm{C}}$ mutations, OS HR was 1.14 (95\% CI: 0.45-2.92) [22]. In the IMPOWER150 study, in the population with KRAS mutations, ABCP showed more benefit in OS and PFS than ACP or BCP; in the KRAS-WT population, comparing with $\mathrm{BCP}$, the improvement in $\mathrm{OS}$ of $\mathrm{ABCP}$ or $\mathrm{ACP}$ was limited[23].

Table 2 Efficacy data of immunotherapy in KRAS mutant population 


\begin{tabular}{|c|c|c|c|c|c|}
\hline Study name & Phase & setting & Arms & $\underset{\mathbf{m}}{\text { No.KRAS }}$ & progress \\
\hline $\begin{array}{l}\text { IMMUNTR- } \\
\text { GET }\end{array}$ & I & $\geq 2$ nd Line & ICIs & 246 & $\begin{array}{c}\text { ORR } 26 \%, \mathrm{~m} \text { PFS3.2 m, } \\
\text { mOS } 13.5 \mathrm{~m}\end{array}$ \\
\hline Keynote042 & III & 1 st Line & $\begin{array}{l}\text { Pem- } \\
\text { bromab }\end{array}$ & 69 & $\begin{array}{c}\text { KRASm vs Wild type: } \\
\text { ORR } 56.7 \% \text { vs } 29.1 \% \text {, } \\
\text { mOS HR 0.42 (95\% CI: } \\
0.22-0.81)\end{array}$ \\
\hline $\begin{array}{l}\text { Checkmate } \\
057\end{array}$ & III & $\geq 2$ nd Line & $\begin{array}{c}\text { Nivoluma } \\
\mathrm{b}\end{array}$ & 62 & $\begin{array}{c}\text { Median OS } 12.2 \mathrm{~m} \text { vs } \\
9.4 \mathrm{~m}, \mathrm{HR} 0.52(95 \% \text { CI } 0.29 \\
0.95)\end{array}$ \\
\hline OAK study & III & $\geq 2$ nd Line & $\begin{array}{l}\text { Atezoli- } \\
\text { zumab }\end{array}$ & 59 & $\begin{array}{c}\text { Median OS } 13.8 \mathrm{~m} \text { vs } \\
9.6 \mathrm{~m}, \mathrm{HR} 0.71(95 \% \mathrm{CI} 0.38 \\
1.34)\end{array}$ \\
\hline $\begin{array}{l}\text { Mata analy- } \\
\text { sis }\end{array}$ & III & $\begin{array}{l}1 \text { st } / 2 \text { nd } \\
\text { Line }\end{array}$ & ICIs & 694 & $\begin{array}{c}\text { KRASm vs Wild type } \\
(\mathrm{OR}=1.51 ; 95 \% \mathrm{CI}: 1.17- \\
1.96 ; \mathrm{P}=0.002)\end{array}$ \\
\hline
\end{tabular}

Table 3 Efficacy data of immunotherapy in KRAS mutant population

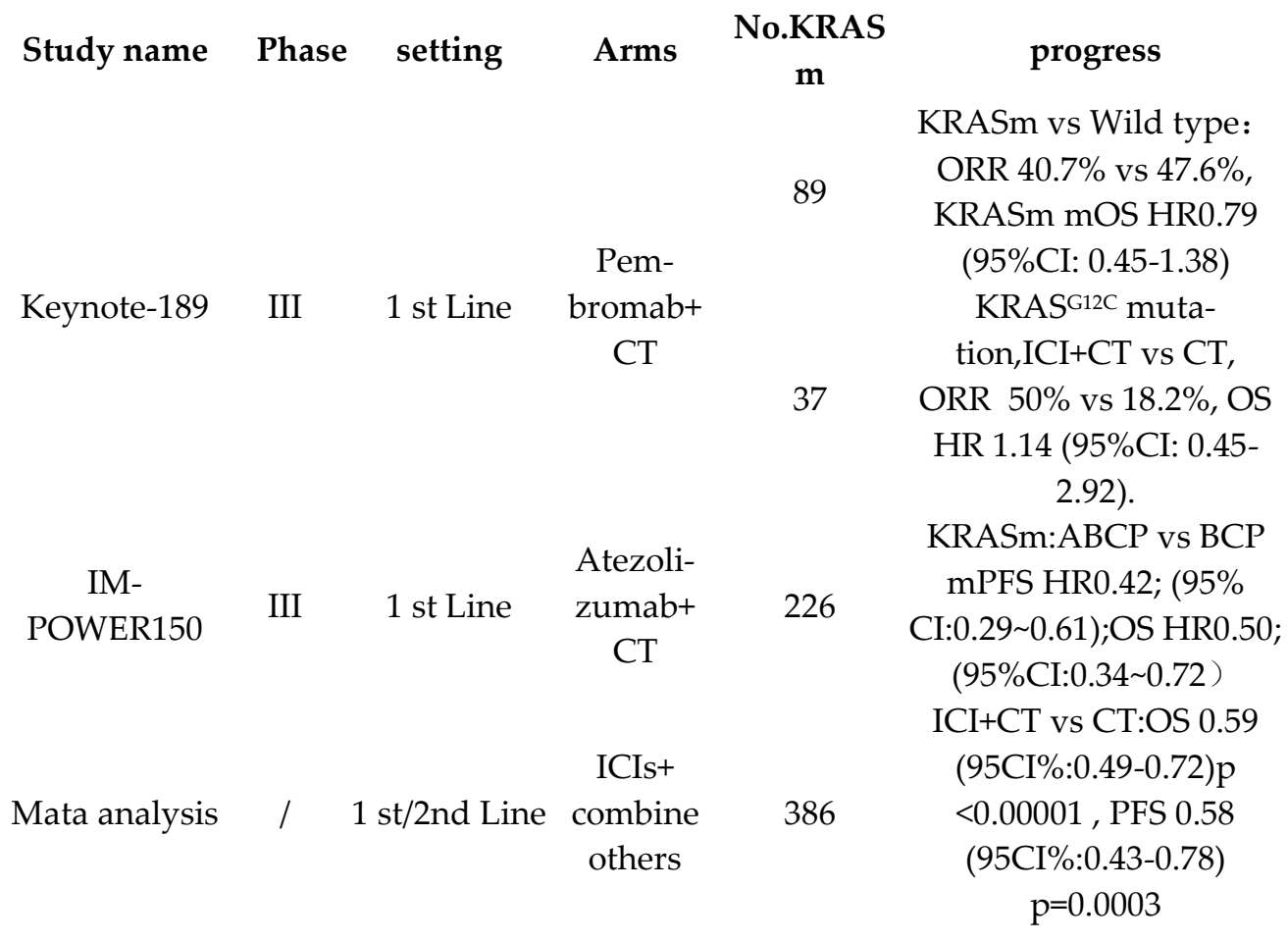

\subsection{The immunomodulatory effect of KRAS mutation gene}

For people with KRAS mutations, immunotherapy is another ray of hope after G12C inhibitors. At the same time, we go through the literature and found that the KRAS mutant population has immunoregulatory properties.

A growing body of evidence shows that KRAS mutation mediates autocrine effects and overlap with the tumor microenvironment (TME) [24]. Pro-inflammatory effects mediated by the activation of transcription factors (STAT3), the production of cytokines (e.g.,IL-6), the activation of NLRP3 inflammasome and the release of chemokines caused by oncogenic KRAS activation. Meanwhile, KRAS-downstream pathways plays an crucial role in shaping the immune microenvironment, where the induction of NF- $k B$ 
activates several cytokines and chemokines, including TNF- $\alpha$, IL1 $\alpha / \beta$, IL-6, CXCL1, 2, 5 and 8; and RAF/MAPK and PI3K also induce IL-10, transforming growth factor $\beta$ (TGF$\beta$ ) and granulocyte-macrophage colony-stimulating factor (GM-CSF) independent of NF- $\kappa$ B57[25].

Yilong et al found that tumor interference (IFNG), programmed death ligand-1(PD-L1), programmed death ligand-1(PD-1), and CD8 expression were higher in KRAS mutant lung cancer[26].There is considerable evidence that oncogenic KRAS signaling induces the expression of a number of immunomodulatory factors, resulting in an immune-suppressive tumor microenvironment. Studies have shown that G12C inhibitors can relieve immunosuppression and reshape the tumor microenvironment. Preclinical studies have shown that $\mathrm{T}$ cell and dendritic cell infiltration increased significantly in tumor-bearing mice treated with GMG510 plus PD-1 for 4 days. At the same time, IFN-r-mediated tumor cell surface antigen increase. The combination of mechanism and animal experiment is expected to realize the combination of immunity and therapy in the future[27].

\section{Outlook}

The KRAS mutant population has been undruggable for 40 years. G12C inhibitors and immunotherapy are the beginning of success. It is necessary to summarize the successful experience of the existing treatment model and explore the direction of the next treatment . To the mechanisms of drug resistance to G12C inhibitors, and the development of other targeted drugs are the first issues to be discussed. On the one hand, To fully understand the heterogeneity of KRAS mutations, the drug resistance mechanism of targeted therapy is the first question to be explored. On the other hand, the immunomodulatory effect of KRAS mutant tumors provides ideas for a combined strategies based on immunotherapy.

There are still many questions to be answered, such as how to overcome resistance to targeted therapy and how to best use combination strategies. Guided by successful clinical studies of G12C inhibitors and immunotherapy, we believe that the future direction may be towards targeting the KRAS-driven proliferative pathway and tumor evasion of the immune system.

\section{References}

1. Helen Adderley , Fiona H Blackhall , Colin R Lindsay. KRAS-mutant non-small cell lung cancer: Converging small molecules and immune checkpoint inhibition. EbioMedicine. 2019 Mar;41:711-716. Doi: 10.1016/j.ebiom.2019.02.049.

2. Graham W Warren, K Michael Cummings. Tobacco and lung cancer: risks, trends, and outcomes in patients with cancer. Am Soc Clin Oncol Educ Book.2013;359-64.Doi: 10.14694/EdBook_AM.2013.33.359.

3. Amanda R Moore, Scott C Rosenberg, Frank McCormick ,et al. Author Correction: RAS-targeted therapies: is the undruggable drugged? Nat Rev Drug Discov.2020 Dec;19(12):902.

4. M Reck,D P Carbone, M Garassino,et al. Targeting KRAS in non-small-cell lung cancer: recent progress and new approaches. Ann Oncol.2021 Sep;32(9):1101-1110.Doi: 10.1016/j.annonc.2021.06.001.

5. Alexander I Spira , Huakang Tu , Shivani Aggarwal ,et al. A retrospective observational study of the natural history of advanced non-small-cell lung cancer in patients with KRAS p.G12C mutated or wild-type disease. Lung Cancer 2021 Sep;159:1-9. Doi: 10.1016/j.lungcan.2021.05.026.

6. Kathryn C Arbour , Emmett Jordan, Hyunjae Ryan Kim, et al. Effects of Co-occurring Genomic Alterations on Outcomes in Patients with KRAS-Mutant Non-Small Cell Lung Cancer. Clin Cancer Res 2018 Jan 15;24(2):334-340. Doi: 10.1158/10780432.CCR-17-1841.

7. Ferdinandos Skoulidis, Lauren A Byers, Lixia Diao,et al. Co-occurring genomic alterations define major subsets of KRASmutant lung adenocarcinoma with distinct biology, immune profiles, and therapeutic vulnerabilities. Cancer Discov 2015 Aug;5(8):860-77. Doi: 10.1158/2159-8290.CD-14-1236. Epub 2015 Jun 11.

8. Alex Friedlaender, Alexander Drilon, Glen J Weiss,et al. KRAS as a druggable target in NSCLC: Rising like a phoenix after decades of development failures. Cancer Treat Rev 2020 Apr;85:101978. Doi: 10.1016/j.ctrv.2020.101978.

9. Jonathan M Ostrem, Ulf Peters, Martin L Sos, James A Well,et al. K-Ras(G12C) inhibitors allosterically control GTP affinity and effector interactions, Nature. 2013 Nov 28;503(7477):548-51. Doi: 10.1038/nature12796. Epub 2013 Nov 20. 
10. Shannon S Zhang, Misako Nagasaka l,et al. Spotlight on Sotorasib (AMG 510) for KRAS G12C Positive Non-Small Cell Lung Cancer. Lung Cancer (Auckl). 2021 Oct 7;12:115-122. Doi: 10.2147/LCTT.S334623.

11. Hong DS, Fakih MG, Strickler JH, et al. KRASG12C inhibition with sotorasib in advanced solid tumors. Presented at: 2020 ESMO Virtual Congress; September 19-21, 2020; Virtual. Abstract 1257O.

12. David S Hong, Marwan G Fakih, John H Strickler, et al. Hong DS, et al. N Engl J Med. 2020;383:1207-1217. N Engl J Med. 2020 Sep 24;383(13):1207-1217.

13. S. Ramalingam,F. Skoulidis,R. Govindan,et al. P52.03 Efficacy of Sotorasib in KRAS p.G12C-Mutated NSCLC with Stable Brain Metastases: A Post-Hoc Analysis of CodeBreaK 100.Doi:https://doi.org/10.1016/j.jtho.2021.08.547.

14. Ferdinandos Skoulidis, Bob T. Li, Ramaswamy Govindan, et al.Overall survival and exploratory subgroup analyses from the phase 2 CodeBreaK 100 trial evaluating sotorasib in pretreated KRAS p.G12C mutated non-small cell lung cancer. Add to Collection.J Clin Oncol 39, 2021 (suppl 15; abstr 9003).10.1200/JCO.2021.39.15_suppl.9003.

15. Ferdinandos Skoulidis,Martin Schuler, Jürgen Wolf,etal.Genomic profiles and potential determinants of response and resistance in KRAS p. ${ }^{\mathrm{G} 12 \mathrm{C}}$-mutated NSCLC treated with sotorasib. Journal of thoracic oncology: official publication of the International Association for the Study of Lung Cancer 16(10):S929-S930.Doi:10.1016/j.jtho.2021.08.184.

16. Gregory J. Riely, Sai-Hong Ignatius Ou, Igor I. Rybkin,et al. KRYSTAL-1: Activity and Preliminary Pharmacodynamic (PD) Analysis of Adagrasib (MRTX849) in Patients (Pts) With Advanced Non-Small- Cell Lung Cancer (NSCLC) Harboring KRAS $^{\mathrm{G} 12 \mathrm{C}}$ Mutation. European Lung Cancer Conference (ELCC), March 25-27, 2021.

17. Chengming Liu, Sufei Zheng, Runsen Jin ,et al. The superior efficacy of anti-PD-1/PD-L1 immunotherapy in KRAS-mutant non-small cell lung cancer that correlates with an inflammatory phenotype and increased immunogenicity. Cancer Lett 2020 Feb 1;470:95-105. Doi: 10.1016/j.canlet.2019.10.027. Epub 2019 Oct 20.

18. Achim Rittmeyer, Fabrice Barlesi, Daniel Waterkamp,et al. Atezolizumab versus docetaxel in patients with previously treated non-small-cell lung cancer (OAK): a phase 3, open-label, multicentre andomized controlled trial. Lancet.2017 Jan 21;389(10066):255-265. Doi: 10.1016/S0140-6736(16)32517-X.

19. Herbst RS, Lopes G, Kowalski DM, et al. Association of KRAS mutational status with response to pembrolizumab monotherapy given as first-line therapy for PD-L1-positive advanced non-squamous NSCLC in KEYNOTE-042. Presented at: 2019 ESMO Immuno-Oncology Congress; December 11-14, 2019; Geneva, Switzerland. Abstract LBA4.

20. J Mazieres, A Drilon, A Lusque ,et al. Immune checkpoint inhibitors for patients with advanced lung cancer and oncogenic driver alterations: results from the IMMUNOTARGET registry. Ann Oncol. 2019 Aug 1;30(8):1321-1328. Doi: 10.1093/annonc/mdz167.

21. Thierry Landre, Gregoire Justeau, Jean-baptiste ASSIE,et al. Anti PD-(L)1 in KRAS mutant advanced nsclcs: A meta-analysis of randomized controlled trials. J Clin Oncol 39, 2021 (suppl 15; abstr 9025). Doi:10.1200/JCO.2021.39.15_suppl.9025.

22. Gadgeel S, Rodriguez-Abreu D, et al. KRAS mutational status and efficacy in KEYNOTE-189: Pembrolizumab (pembro) plus chemotherapy (chemo) vs placebo plus chemo as first-line therapy for metastatic non-squamous NSCLC. Presented at: 2019 ESMO Immuno-Oncology Congress; December 11-14, 2019; Geneva, Switzerland. Abstract LBA5.

23. H. West1, F. Cappuzzo, M. Reck,et al. IMpower150: A post hoc analysis of efficacy outcomes in patients with KRAS, STK11 and KEAP1 mutations. Annals of Oncology (2020) 31 (suppl_4): S754-S840. 10.1016/annonc/annonc283.

24. Hadrien G Golay, David A Barbie,et al. Targeting cytokine networks in KRAS-driven tumorigenesis. Expert Rev Anticancer Ther. 2014 Aug;14(8):869-71.Doi: 10.1586/14737140.2014.928596. Epub 2014 Jun 13.

25. Véronique Baud, Michael Karin. Is NF-kappaB a good target for cancer therapy? Hopes and pitfalls. Nat Rev Drug Discov. 2009 Jan;8(1):33-40. Doi: 10.1038/nrd2781.

26. Xu-Chao Zhang, Jun Wang, Guo-Guang Shao,et al. Comprehensive genomic and immunological characterization of Chinese non-small cell lung cancer patients. Nat Commun. 2019 Apr 16;10(1):1772. Doi: 10.1038/s41467-019-09762-1.

27. Jude Canon, Karen Rex , Anne Y Saiki,et al. The clinical KRAS(G12C) inhibitor AMG 510 drives anti-tumour immunity. Nature. 2019 Nov;575(7781):217-223. Doi: 10.1038/s41586-019-1694-1. Epub 2019 Oct 30. 